\title{
Erratum to: Facial Fabrication of Biomimetic Water Pinning Microstructures on Polyethylene Surfaces with Robust Superhydrophobic Wetting State
}

\author{
Yuzhu $\mathrm{Li}^{1,2}$, Hang Gao ${ }^{1,2^{*}}$, Yunhai $\mathrm{Ma}^{3}$, Donghui Chen ${ }^{3}$ \\ 1. School of Mechatronics Engineering, Shenyang Aerospace University, Shenyang 110136, China \\ 2. Key Laboratory of Fundamental Science for National Defense of Aeronautical Digital Manufacturing Process, \\ Shenyang 110136, China \\ 3. Key Laboratory for Bionic Engineering, Ministry of Education, Jilin University, Changchun 130022, China
}

Copyright (C) Jilin University 2020.

Erratum to: J Bionic Eng (2020) 17(4): 644-651

https://doi.org/10.1007/s42235-020-0052-1

The title was incorrect in the published paper and should be revised as "Facile Fabrication of Biomimetic Water Pinning Microstructures on Polyethylene Surfaces with Robust Superhydrophobic Wetting State".

The online version of the original article can be found at https://doi.org/10.1007/s42235-020-0052-1

\footnotetext{
*Corresponding author: Hang Gao

E-mail: saugaohang@163.com
} 